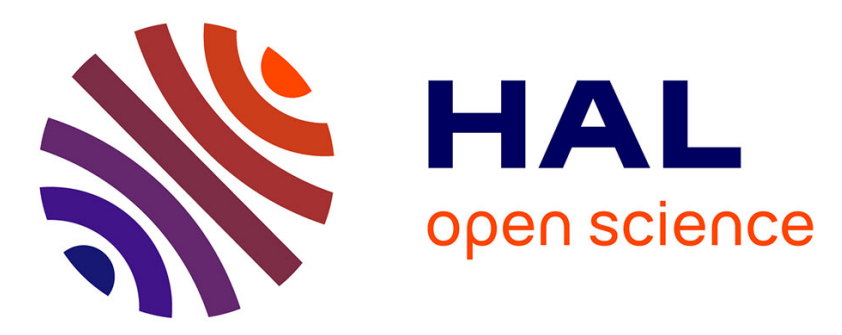

\title{
The importance of specific mechanical energy during twin screw extrusion of organoclay based polypropylene nanocomposites
}

\author{
Trystan Domenech, Edith Peuvrel-Disdier, Bruno Vergnes
}

\section{- To cite this version:}

Trystan Domenech, Edith Peuvrel-Disdier, Bruno Vergnes. The importance of specific mechanical energy during twin screw extrusion of organoclay based polypropylene nanocomposites. Composites Science and Technology, 2013, 75, pp.7-14. 10.1016/j.compscitech.2012.11.016 . hal-00769978

\section{HAL Id: hal-00769978}

https://hal-mines-paristech.archives-ouvertes.fr/hal-00769978

Submitted on 13 Apr 2017

HAL is a multi-disciplinary open access archive for the deposit and dissemination of scientific research documents, whether they are published or not. The documents may come from teaching and research institutions in France or abroad, or from public or private research centers.
L'archive ouverte pluridisciplinaire HAL, est destinée au dépôt et à la diffusion de documents scientifiques de niveau recherche, publiés ou non, émanant des établissements d'enseignement et de recherche français ou étrangers, des laboratoires publics ou privés. 


\title{
The importance of specific mechanical energy during twin screw
}

\section{extrusion of organoclay based polypropylene nanocomposites}

Trystan Domenech, Edith Peuvrel-Disdier, Bruno Vergnes*

MINES ParisTech, Centre de Mise en Forme des Matériaux (CEMEF),

UMR CNRS 7635, BP 207, 06904 Sophia-Antipolis Cedex (France)

*Corresponding author.

E-mail address: bruno.vergnes@mines-paristech.fr (B. Vergnes).

\begin{abstract}
This study demonstrates how the specific mechanical energy (SME) can be used to describe the influence of extrusion parameters such as screw rotation speed, feed rate and barrel temperature on clay dispersion in organoclay (OMMT) based polypropylene nanocomposites. These materials were prepared by a melt mixing masterbatch process via twin screw extrusion with a wide range of processing conditions. Maleated polypropylene (PP-g-MA) was used as a compatibilizer to allow clay exfoliation. Characterization of the morphological evolution along the extrusion profile revealed that microscale dispersion primarily happens in the melting zone, whereas continuous exfoliation is observed all along the kneading zones, up to the die exit. The results indicate that exfoliation in the kneading zones is mainly issued from clay tactoids and small aggregates with characteristic size inferior to $10 \mu \mathrm{m}$, emphasizing the crucial role of primary microscale dispersion on the final structure and properties of the nanocomposites. Relevant quantitative prediction of the multiscale dispersion state along the extrusion profile was obtained using the melt state SME as unique parameter.
\end{abstract}

Keywords: A. Nanocomposites, A. Polymer-matrix composites (PMCs), D. Rheology, E. Extrusion, Dispersion 


\section{Introduction}

Reinforcement of polymer matrix by the addition of nanoclay particles is an efficient strategy to extend polymers' range of applications by enhancing their mechanical $[1,2]$, thermal $[3,4]$ and barrier properties [5,6]. Nanostructuring of these hybrid materials is obtained by intercalation or exfoliation of the native stacked clay platelets by the macromolecules $[3,7,8]$. Melt processing has revealed to be a very suitable route for nanocomposites production as it benefits flexible processability of continuous mixing processes and does not require the use of solvents, which is advantageous from an environmental and economical point of view.

In the case of widely used polyolefin matrices such as polypropylene, the grafting of polar groups such as maleic anhydride along polypropylene backbone turns out an effective approach to improve polymer-clay affinity and allows the formation of intercalated/exfoliated polypropylene based nanocomposites [9-11].

Besides compatibilization concern, it is important to understand how processing conditions affect the structure of nanocomposites during melt mixing step in order to establish processing strategies which could lead to highly exfoliated PP based nanocomposites. Out of the massive literature dealing with nanocomposites, few references discuss the influence of processing conditions on the resulting nanocomposites' structure [2,10,12-18]. An important aspect of the melt processing approach is to fulfill a dispersive mixing, i.e. to break the native organoclay agglomerates down to individual layers. Several studies revealed that the state of dispersion of the nanocomposites is sensitive to both shear intensity and residence time [10,12,15-19]. Furthermore, quantitative characterization of the overall dispersion state is quite challenging as nanocomposites can be highly polydisperse systems. X-ray diffraction (XRD) and transmission electron microscopy (TEM) are widely used to 
describe nanoscale organization. However, these characterization techniques provide a local insight and cannot be representative of the whole range of the dispersion state $[20,21]$. On the other hand, melt rheology is very sensitive to the nanostructure $[10,22-$ 29] and has the advantage to probe macroscopic volume properties of the nanocomposites samples.

Beyond of fundamental interest, it is also crucial to understand the mechanisms of clay dispersion from its natural agglomerate form to exfoliated nanostructure during the extrusion process. Modularity of twin screw extruders enables the use of numerous types of elements (conveying screw, back flowing screw, mixers, kneaders, etc) which can be combined with countless possibilities. The design of screw profile will affect flow properties within the extruder and local parameters like filling ratio, shear rate, temperature and residence time. Consequently, it will also influence distribution and dispersion of the clay within the matrix. In a previous study [30], we showed how the influence of processing conditions on the final structure of extruded nanocomposites can be described by a single parameter, using the specific mechanical energy (SME). The latter represents the level of energy per mass unit that is transferred to the material by mechanical input during extrusion. In the present article, we emphasize on the evolution of dispersion state along the extrusion profile by investigating the role of local SME contribution and dispersion mechanisms of the organoclay filler, using identical nanocomposite formulation [30]. For this purpose, dead-stop experiments were carried out after reaching stationary conditions in order to sample materials from various zones along the extrusion profile. However, the lack of information about local extrusion parameters during melt processing raises a problem in the establishment of processingstructure relationships along the screw profile. To overcome this limitation, we used a 
flow simulation software (Ludovic ${ }^{\circledR}$ ) to calculate local extrusion parameters along the screw profile [31] and confront experimental results with empiric laws based on SME.

\section{Materials and methods}

\subsection{Materials}

The polypropylene (PP) used in this study was a semi-crystalline injection grade isotactic homopolymer produced by LyondellBasell under the reference Moplen HP400R. It had a density of $0.905 \mathrm{~g} / \mathrm{cm}^{3}\left(25^{\circ} \mathrm{C}\right)$, a melting temperature of $165^{\circ} \mathrm{C}$ and a $25 \mathrm{~g} / 10$ min melt flow index $\left(230^{\circ} \mathrm{C}, 2.16 \mathrm{~kg}\right)$. A montmorillonite based organoclay (OMMT, Dellite ${ }^{\circledR} 67 \mathrm{G}$ ) in powder form with a density of $1.7 \mathrm{~g} / \mathrm{cm}^{3}$ was kindly provided by Laviosa Chimica Mineraria. This grade of organoclay featured $48 \mathrm{wt} \%$ of dimethyldehydrogenated-tallow quaternary ammonium as organic modifier and a cation exchange capacity of 115 meq/100 g. Polypropylene grafted with maleic anhydride (PPg-MA, Eastman ${ }^{\mathrm{TM}}$ G-3015) was purchased from Eastman Chemical Company and used as a compatibilizer. This grade had a maleic anhydride content of $3.1 \mathrm{wt} \%$, a density of $0.913 \mathrm{~g} / \mathrm{cm}^{3}$ and a melting temperature of $162^{\circ} \mathrm{C}$.

\subsection{Compounding of organoclay based PP nanocomposites}

Nanocomposites were prepared via a masterbatch process [13,17]. A masterbatch with a PP-g-MA to OMMT ratio of 2:1 was compounded at the Danish Technological Institute, as described elsewhere [32], with following formulation: $40 \mathrm{wt} \% \mathrm{PP}, 40 \mathrm{wt} \%$ PP-g-MA and 20 wt\% OMMT. In a second step, the masterbatch was diluted into the PP matrix to produce PP/PP-g-MA/OMMT nanocomposites with respective weight fractions of 85/10/5 using a ThermoFisher Rheomex PTW24 co-rotating twin screw extruder with laboratory scale modular screw profile design (diameter $D=24 \mathrm{~mm}$, length to diameter ratio $L / D=40$ ). The screw configuration is described in Fig. 1 . The temperature was set identically for all barrel elements, except for the first one which 
was regulated at $80^{\circ} \mathrm{C}$. An appropriate mixture of $\mathrm{PP}$ and masterbatch pellets was tumbled mixed and then fed into the hopper using a gravimetric feeder. Once extrusion conditions have reached steady state, extruded strands were pelletized. Nanocomposites were prepared for a wide range of processing conditions, depending on screw rotation speed $N$, feed rate $Q$ and regulated barrel temperature $T_{\text {reg }}$, as listed in Table 1 . Each of these three parameters was varied individually to assess its effect on the resulting state of dispersion and properties of the nanocomposites. For each extrusion condition, the drive motor torque $\tau$ was recorded. The specific mechanical energy (SME, in $\mathrm{kWh} / \mathrm{t}$ ) was then calculated using Eq. (1) which involves motor power $\left(P_{\text {motor }}=11000 \mathrm{~W}\right)$ and maximum values of drive motor torque $\left(\tau_{\max }=180 \mathrm{Nm}\right)$ and screw rotation speed $\left(N_{\max }\right.$ $=1000 \mathrm{rpm})$ :

$S M E=\frac{P_{\text {motor }}}{\tau_{\max } \cdot N_{\max }} \cdot \frac{\tau \cdot N}{Q}$

Extruded nanocomposites pellets were collected and dried in a vacuum oven at $80^{\circ} \mathrm{C}$ during $15 \mathrm{~h}$, prior to be formed into disk-shaped samples [30] for further material characterization.

\subsection{Dead-stop experiments}

The Rheomex extruder is equipped with a clam-shell barrel system which can be opened to allow access to the screw profile. Dead-stop experiments were carried out by suddenly stopping the extruder once stationary conditions were reached (typically after 10 minutes). Then, barrel was cooled down to $100^{\circ} \mathrm{C}$ by internal water flow in approximately 15 minutes, before opening the barrel. Few quantities of materials (between 2 and $4 \mathrm{~g}$ ) were collected in the material-filled zones of the screw profile, i.e. in the kneading elements. These sampling zones were indexed from A to J, as indicated 
on Fig. 1. This sampling was carried out for screw rotation speeds of 100, 300 and 500 rpm at constant feed rate and barrel temperature of $3 \mathrm{~kg} / \mathrm{h}$ and $180^{\circ} \mathrm{C}$, respectively.

\subsection{Characterization}

In order to estimate the efficiency of dispersive mixing during extrusion, multi-scale morphological characterization was performed on each sample. The presence of undispersed clay agglomerates was detected using a Philips XL30 scanning electron microscope (SEM) as described elsewhere [30]. Particle analysis was carried out from the SEM pictures using Visilog image analysis software. This step allowed to quantify microscale dispersion through the area ratio $A_{r}$ (\%) defined by Eq. (2), where $A_{M M T}$ is the area occupied by residual clay agglomerates and $A_{0}$ is the analyzed section area (equals to $17.2 \mathrm{~mm}^{2}$ ). Clay particles with equivalent diameter inferior to $10 \mu \mathrm{m}$ were not taken into account. The quality of microscale dispersion is thus augmented when $A_{r}$ decreases.

$$
A_{r}=100 \cdot \frac{\sum A_{M M T}}{A_{0}}
$$

Local nanoscale organization was observed from ultra-thin sections using a Philips CM12 transmission electron microscope (TEM) [30]. The mean basal spacing $d_{001}$ of the samples was measured using a Philips Xpert' Pro X-ray diffractometer (XRD) in order to assess the extent of intercalation [30].

Rheological measurements were used to quantify the extent of exfoliation. Small amplitude oscillatory shear (SAOS) measurements were performed on a straincontrolled ARES rheometer (TA Instruments). Parallel plate geometry with $25 \mathrm{~mm}$ diameter and $1 \mathrm{~mm}$ gap was used. All rheological tests were conducted in the molten state with a fixed temperature of $180^{\circ} \mathrm{C}$ under nitrogen environment, using compressed disk samples. Prior to frequency sweep tests, samples were equilibrated after loading in the rheometer at $180^{\circ} \mathrm{C}$ for one-half hour, similarly to protocols previously proposed by 
other authors $[10,24]$. Frequency sweep tests were carried out from $10^{2}$ to $10^{-2} \mathrm{rad} / \mathrm{s}$ in angular frequency. Shear strain did not exceed $1 \%$ to ensure these tests were conducted within the linear viscoelastic range and a new sample was used for each rheological test. Above their percolation threshold, nanocomposites were reported to display a nonterminal low frequency response which is indicative of a solid-like behavior, with the appearance of a storage modulus ( $\left.G^{\prime}\right)$ plateau and a significant increase of the complex viscosity $\left(\left|\eta^{*}\right|\right)$ in the low frequency regime [10,22-24]. This solid-like behavior of nanocomposites has been attributed to the existence of a three-dimensional percolation network formed by anisotropic clay platelets within the matrix [22-24]. Lertwimolnun and Vergnes [10] proposed using a Carreau-Yasuda model combined with a yield stress term (Eq. 3) to describe the frequency dependence of nanocomposites' complex viscosity $\left|\eta^{*}\right|$, where $\omega$ is the angular frequency, $\sigma_{0}$ is the melt yield stress, $\eta_{0}$ is the zero-shear viscosity, $\lambda$ is characteristic relaxation time, $a$ is the Yasuda parameter and $m$ is the shear-thinning index:

$\left|\eta^{*}(\omega)\right|=\frac{\sigma_{0}}{\omega}+\eta_{0} \cdot\left[1+(\lambda \cdot \omega)^{a}\right]^{(m-1) / a}$

Moreover, $\sigma_{0}$ has been shown to efficiently characterize the level of exfoliation $[10,15,16,30]$.

\subsection{Flow modeling}

Simulations of flow conditions along the co-rotating twin screw extruder were performed using Ludovic $^{\odot}$ software, which enables the calculation of the main local flow variables like shear rate, filling ratio, melt temperature, pressure, residence time and dissipated energy, from melting zone to die exit [31]. Numerous experimental validations in compounding (filler dispersion, reactive extrusion), optimization and scale-up applications have shown that $\operatorname{Ludovic}^{\circledR}$ offers consistent description of the flow 
conditions in twin screw extrusion processes [33,34]. These simulations were carried out to establish relationships between nanocomposites structure and processing parameters along the screw profile, where local physical values cannot be precisely measured (or measured at all) during processing. Rheological data (flow curves) of the PP/PP-g-MA matrix were used for those calculations. Indeed, for the range of shear rates encountered in extrusion (10-100 $\left.\mathrm{s}^{-1}\right)$, there is quite no difference in viscosity between matrix and nanocomposite.

\section{Results and discussion}

\subsection{Morphology of native OMMT and masterbatch}

Dellite $^{\odot} 67 \mathrm{G}$ is an off-white powder, composed of agglomerated layered silicate with characteristic size comprised between 10 and $40 \mu \mathrm{m}$ [30]. These bundles of stacked OMMT layers present van-der-Waals interactions leading to important cohesive forces, which must be overcome by the applied shear stresses during melt mixing

process in order to reach nanoscale dispersion. Basal spacing $d_{001}$ of the Dellite ${ }^{\odot} 67 \mathrm{G}$ was found to be $3.37 \mathrm{~nm}$ by XRD measurements.

SEM study of the masterbatch revealed the existence of OMMT agglomerates within the matrix, with equivalent diameter ranging from 10 to $170 \mu \mathrm{m}$ (Fig. 2a), leading to an area ratio $A_{r}$ of $12.8 \%$. The presence of OMMT bundles exceeding $40 \mu \mathrm{m}$ in equivalent diameter indicates that agglomeration of the OMMT powder occurred during the masterbatch compounding step. TEM observations showed the presence of tactoids containing 3 to 5 stacked layers, as well as individualized platelets which is a sign of partial exfoliation (Fig. 2b). XRD experiments provided a basal spacing $d_{001}$ of $3.44 \mathrm{~nm}$, denoting a partial intercalation of the tactoids present within the masterbatch.

\subsection{Influence of processing conditions on melt state SME and resulting structure}


The mechanical energy transferred to the material during the extrusion process depends on various physical parameters such as shear intensity, residence time and melt temperature. It is therefore important to understand how processing parameters like screw rotation speed, feed rate and barrel temperature affect the SME prior to the establishment of relationships between the SME and the dispersion state. Since the mixing process takes place in the melt state, it seems appropriate to consider that the energy consumed by the solid conveying and the melting on the first part of the screw profile (Fig. 1) does not contribute to the OMMT dispersion. Ludovic ${ }^{\odot}$ simulations $^{2}$ allow to calculate the SME dissipated in the melt state $\left(S M E_{\text {melt }}\right)$ which strongly depends on processing conditions. The increase of screw rotation speed induces torque reduction due to shear thinning behavior of the polymeric matrix, as well as a lower filling ratio of the extruder and important viscous heating. Despite torque decrease, screw speed augmentation results in a linear increase of $S M E_{\text {melt }}$. On the other hand, feed rate augmentation simultaneously leads to torque increase (as expected from higher filling ratio) and residence time reduction, giving rise to $S M E_{\text {melt }}$ decrease. Furthermore, $S M E_{\text {melt }}$ is found to lessen linearly with barrel temperature. This trend is related to torque reduction caused by the decrease of the matrix viscosity.

Here, we show that $S M E_{\text {melt }}$ is a key descriptor of processing influence on OMMT multiscale dispersion, similarly to the total SME defined by (Eq. 1) [30]. Plot of the area ratio as a function of $S M E_{\text {melt }}$ is represented on Fig. 3a. Variations of processing conditions through the settings of screw speed, feed rate and barrel temperature lead to a mastercurve that underlies the predominance of $S M E_{\text {melt }}$ on microscale dispersion. The increase of $S M E_{\text {melt }}$ from its lowest to its highest value in the investigated range leads to a reduction of the area ratio by a factor of seven, linked to a significant decrease in number and size range of OMMT agglomerates. Microscale dispersion is thus enhanced 
when processing conditions are set to maximize $S M E_{\text {melt }}$, at least in the studied range. The area ratio was found to scale with $S M E_{\text {melt }}$ as a decreasing power law. Similar correlation has been recently found by Villmow et al. [35], using the experimentally measured SME, in the case of polycaprolactone/multi-walled carbon nanotube composites, where the nanotubes were also introduced into the extruder as agglomerated powder.

The effect of $S M E_{\text {melt }}$ on the exfoliation level is illustrated on Fig. 3b. As said before, rheological analysis allows to determine the yield stress $\sigma_{0}$ which is used as a quantitative measurement of the extent of exfoliation. Fig. 3b shows that a mastercurve is built when the yield stress is represented as a function of $S M E_{m e l t}$, whatever the processing conditions. At first, the yield stress grows linearly with $S M E_{\text {melt }}$ up to a value close to $360 \mathrm{kWh} / \mathrm{t}$. Above this critical $S M E_{\text {melt }}$ value, the yield stress stabilizes at a plateau value, suggesting that no further exfoliation seems to occur. Médéric et al. [18] found a similar result with polyamide 12/OMMT nanocomposites processed in an internal mixer, with much lower critical SME value (around $140 \mathrm{kWh} / \mathrm{t}$ in experimental value). This may suggest that the critical SME depends on the polymer-clay affinity since exfoliation is known to be much easier in a polar PA matrix rather than in PP $[9,12]$. The saturation of the extent of exfoliation reveals a certain limit of the influence of processing conditions on nanoscale dispersion. This phenomenon is observed for the highest screw rotation speeds, where important shear thinning and high melt temperature can soften the shear stresses and thus limit layer delamination. Moreover, XRD measurements indicate a partial collapse of the interlayer spacing when screw rotation speed exceeds $500 \mathrm{rpm}$ (Table 1), what also might inhibit exfoliation. Considering the temperature range $\left(210-260{ }^{\circ} \mathrm{C}\right)$ and the mean residence times (90-110 s), this could be due to thermal degradation of the surfactant, as often evoked in the literature, but desorption of the surfactant molecules from the interlayer spacing could also be a possible mechanism.

TEM observations shown in Fig. 3b highlight the simultaneous presence of partially oriented tactoids and single layers. Augmentation of the yield stress corresponds to a reduction in tactoids thickness, as well as an increase in individual layers number and a shortening of interparticle distances, indicating an enhancement of nanoscale dispersion 
which confirms the trend depicted by rheological measurements. We recently showed that this raise in exfoliation level led to higher stiffness of the nanocomposites in the semicrystalline state [30].

\subsection{Morphological evolution along the extrusion profile}

\subsubsection{Multiscale observations}

The progress of OMMT dispersion during extrusion processing was investigated by following the structural features of the hybrid material along the extrusion profile. This analysis was carried out for screw rotation speeds of 100, 300 and $500 \mathrm{rpm}$ at constant feed rate $(3 \mathrm{~kg} / \mathrm{h})$ and barrel temperature $\left(180^{\circ} \mathrm{C}\right)$. Fig. 4a shows typical SEM pictures of the samples collected along the screw profile after processing $(N=500 \mathrm{rpm}, Q=3$ $\left.\mathrm{kg} / \mathrm{h}, T_{\text {reg }}=180^{\circ} \mathrm{C}\right)$. A significant reduction in size of the OMMT agglomerates is observed from zone A to B, i.e. the melting zone. In fact, even the frontier between PP and masterbatch pellets can be distinguished on the zone A sample as the melting of polymers is incomplete at this stage of the extrusion process. Afterwards, no meaningful evolution of the microscale dispersion of the OMMT can be observed from zone B to the die exit. These observations point out that microscale dispersion mainly happens in the melting zone, since most of the OMMT agglomerates present afterwards remained undispersed within the matrix.

Evolution of nanoscale dispersion along the extrusion profile was also monitored using TEM, as illustrated in Fig. 4b for the same processing conditions. The increase in tactoids and single platelets number together with the reduction of tactoids thickness indicates an enhancement of the exfoliation level along the extrusion profile. One can note that the orientation of clay particles (tactoids and platelets) becomes more pronounced as the material flows towards the die exit, which could be attributed to a progressive flow induced alignment of anisotropic clay particles [36,37]. 


\subsubsection{Rheological analysis}

Linear viscoelastic behavior of PP/PP-g-MA matrix and nanocomposites samples from various zones of the extrusion profile is represented in Fig. 5. The matrix exhibits a classical Carreau-Yasuda behavior, whereas nanocomposites show a low frequency $G$ ' plateau and yield stress behavior. Rheological data were fitted using the CarreauYasuda with yield stress model (Eq. (3)) by adjustment of the five parameters. As shown on Fig. 5b, an excellent agreement is observed between experimental data and fitted curves, confirming that Eq. (3) provides a very accurate description of the nanocomposites rheological behavior. The increase of the exfoliation level intensifies the solid-like behavior of the nanocomposites and leads to a yield stress augmentation, which can thus be used as a quantitative parameter to measure the extent of exfoliation at fixed clay content $[10,28]$. Here, the low frequency $G^{\prime}$ plateau becomes increasingly marked and reaches higher values as material progresses towards the die exit, suggesting the development of a long scale network formed by anisotropic clay platelets through progressive exfoliation along the screw profile, in accordance with local TEM observations. Augmentation of screw speed results in a substantial acceleration of nanoscale morphological development along the extrusion profile, as shown on Fig. 6b.

\subsubsection{Correlation between dispersion progress and local SME}

In order to explore the influence of processing on the progressive nanocomposite formation along the screw profile, flow parameters of the twin-screw extrusion process were calculated using Ludovic ${ }^{\circledR}$ software. Excellent agreement between experimental measurements and Ludovic $^{(}$results was found in terms of residence time, melt temperature at the die exit, die pressure and SME, confirming the relevance of these simulations. Fig. 7 shows the evolution of $S M E_{\text {melt }}$ along the screw profile for several 
screw rotation speeds. It clearly appears that energy is mainly dissipated in the zones containing blocks of kneading discs, whereas conveying elements show insignificant dissipation. The rise in screw rotation speed magnifies the increase of $S M E_{\text {melt }}$ along the screw profile, in accordance with the results presented in section 3.2. In this selected range of processing conditions, the area ratio and melt yield stress of the samples collected at the die exit can be described as a function of $S M E_{\text {melt }}$ using Eq. (4) and (5), respectively, where $\alpha=47.14 \mathrm{kWh}^{\mathrm{n}} / \mathrm{t}^{\mathrm{n}}, \beta=4.78 \mathrm{~Pa} . \mathrm{t} / \mathrm{kWh}$ and $n=0,7321$ :

$$
\begin{aligned}
& A_{r}=\alpha \cdot S M E_{\text {melt }}^{-n} \\
& \sigma_{0}=\beta \cdot S M E_{\text {melt }}
\end{aligned}
$$

These results correspond to the trends shown in Figs. 4 and 5 for the same range of processing conditions.

Based on calculations of local $S M E_{\text {melt }}$ values via Ludovic ${ }^{\circledR}$ simulations, Eq. (4) and (5) were used to calculate the area ratio and melt yields stress along the screw profile. Comparisons of calculated values (dashed lines) with experimental values (symbols) are presented in Fig. 6. A fair agreement is obtained, despite some deviations can be noted concerning the variations of the area ratio in zones A through $\mathrm{C}$, where agglomerate dispersion was found to be more drastic. Nevertheless, these calculations lead to a satisfactory description of the multiscale OMMT dispersion state development along the extrusion profile, without any adjustment parameter.

These results confirm that microscale dispersion of OMMT principally takes place in the melting zone, whereas nanoscale dispersion appears to follow a progressive development along the screw profile. The dispersion efficiency at both micro and nanoscale is clearly favored by the increase of screw rotation speed, at least up to 500 rpm, in accordance with the results presented in section 3.2. 


\subsubsection{Dispersion mechanisms}

Understanding of OMMT dispersion mechanisms involved during extrusion is a key step towards melt processing optimization. Plot of the melt yield stress as a function of the area ratio presented in Fig. 8a illustrates the dispersion regimes. For extrusion zones A and B (white region in Fig. 8a), a significant reduction of the area ratio occurs while the melt yield stress is almost unaffected. This indicates the preponderance of OMMT agglomerate fragmentation into smaller particles with characteristic size inferior to 10 $\mu \mathrm{m}$, as illustrated in Fig. 8b. Furthermore, the influence of shear stress on microscale dispersion suggests a rupture mechanism [38,39], which could explain why the dispersion of OMMT agglomerates primarily occurs in the melting zone since shear stresses are maximum in this part of the extrusion profile. The influence of feed rate on the area ratio (Fig. 3a) reveals the sensitivity of microscale dispersion to residence time which could be the signature of an erosion-like mechanism, previously observed in shear flows for other fillers like carbon black [38], silica [40] and carbon nanotubes [39].

A change of dispersion regime is observed after the melting zone (grey region in Fig. 8a), where a substantial rise of the melt yield stress is accompanied by a slight decrease of the area ratio from zone B to the die exit, highlighting the preponderance of OMMT exfoliation. Since the area ratio is nearly unchanged after the melting zone, exfoliation of the layers must come from clay particles with characteristic size less than $10 \mu \mathrm{m}$, i.e. aggregates and tactoids. The TEM micrograph presented on Fig. 8c supports this hypothesis and reveals that OMMT platelets seem to be peeled off from the surface of aggregated stacks. Similar observation of peel apart mechanism was reported by

Dennis et al. [12] in the case of melt processed organoclay based nylon 6 nanocomposites. Thereby, OMMT dispersion by melt mixing in twin screw extrusion 
can be described as a two-step process. First, microscale dispersion takes place in the melting zone by the fragmentation of agglomerates into smaller tactoids and aggregates. However, this step results in a certain amount of undispersed agglomerates whose number and size depend on processing conditions. The second step is a progressive delamination of the montmorillonite layers, which takes place in the kneading zones up to the die exit. The latter strongly depends on processing conditions and may only be possible in the case of intercalation of the PP-g-MA compatibilizer between stacked layers $[9,10]$.

\section{Summary and conclusions}

This article offers in-depth discussion about the relationships between processing and multiscale structure of PP/PP-g-MA/OMMT nanocomposites prepared by twin screw extrusion via a masterbatch dilution method. Melt processed nanocomposites revealed to be highly polydisperse systems, exposing the concomitant presence of OMMT individual layers, tactoids and agglomerates. The microscale feature of nanocomposites is rarely probed in the structural analysis that can be found in the literature, where the focus is mainly set on local nanoscale organization through XRD and TEM analysis. Nonetheless, this aspect is crucial since the presence of agglomerates might act as structural defects which could be related to the failure of mechanical properties enhancement predictions.

Development of the multiscale dispersion state of the nanocomposites during melt processing was determined thanks to samples collection along the screw profile. Numerical simulations using Ludovic ${ }^{\circledR}$ software allowed a quantitative description of the dispersion state development along the extrusion profile based on melt state SME calculations. The melting zone revealed to be crucial regarding microscale dispersion, as reduction in number and size of the OMMT agglomerates mainly takes place in this 
part of the screw profile. The presence of residual agglomerates after the melting zone underlies the importance of microscale structural characterization of the extruded materials. A progressive increase of the extent of exfoliation was found to arise from the SME contribution of kneading elements on the rest of the screw profile. Additionally, this work gives an insight of the OMMT dispersion mechanisms involved during extrusion. In a first step, agglomerates are sharply broken down into smaller aggregates and tactoids by the mean of rupture and erosion mechanisms. These smaller clay particles are then exfoliated through a peel-like mechanism which is favored by the rise of shear intensity up to a certain limit.

Ultimately, SME appears to be a key parameter of melt processing with regard to the structure and properties of nanocomposites, even though it might not be the only influential parameter since the effect of screw profile design was not reported here. In terms of applications, results have shown that structuring of nanocomposites using twin screw extrusion can be optimized by working under high SME conditions until a critical limit beyond which exfoliation can no longer be enhanced by the processing conditions. Existence of critical phenomenon concerning the extent of exfoliation may indicate a limited effect of shear intensity on layer individualization. As a consequence, use of elongational strain type of elements in the kneading zone of the screw configuration might be a solution to extend the processing limits highlighted in this study.

\section{Acknowledgement}

Authors thank Stephanie Bonny, Thierry Colin, Walid Bahloul, Nathalie Bozzolo and Gabriel Monge (CEMEF, France) for their precious contributions to this work, as well as Aleksey Drozdov (DTI, Denmark) for gently providing the masterbatch. Financial support by the European Commission through FP7 project Nanotough-213436 is gratefully acknowledged. 


\section{References}

[1] Kojima Y, Usuki A, Kawasumi M, Okada A, Fukushima Y, Kurauch T, Kamigaito O. Mechanical properties of nylon 6-clay hybrid. J Mater Res 1993; 8:1185-9.

[2] Cho J, Paul D. Nylon 6 nanocomposites by melt compounding. Polymer 2001; 42:1083-94.

[3] Giannelis EP. Polymer layered silicate nanocomposites. Adv Mater 1996; 8:29-35.

[4] Zanetti M, Camino G, Canavese B, Morgan AB, Lamelas FJ, Wilkie CA. Fire retardant halogenantimony-clay synergism in polypropylene layered silicate nanocomposites. Chem Mater 2002; 14:18993.

[5] Messersmith PB, Giannelis EP. Synthesis and barrier properties of poly( $\varepsilon$-caprolactone)-layered silicate nanocomposites. J Polym Sci Part A - Polym Chem 1995; 33:1047-57.

[6] Sherman LM. Nanocomposites: A little goes a long way. Plast Technol 1999; 45:52-7.

[7] Vaia RA, Ishii H, Giannelis E. Synthesis and properties of two-dimensional nanostructures by direct intercalation of polymer melts in layered silicates. Chem Mater 1993; 5:1694-6.

[8] Vaia R, Jandt K, Kramer E, Giannelis E. Kinetics of polymer melt intercalation. Macromolecules 1995; 28:8080-5.

[9] Kawasumi M, Hasegawa N, Kato M, Usuki A, Okada A. Preparation and mechanical properties of polypropylene-clay hybrids. Macromolecules 1997; 30:6333-38.

[10] Lertwimolnun W, Vergnes B. Influence of compatibilizer and processing conditions on the dispersion of nanoclay in a polypropylene matrix. Polymer 2005; 46:3462-71.

[11] Kim DH, Fasulo P, Rodgers W, Paul DR. Structure and properties of polypropylene-based nanocomposites: Effect of PP-g-MA to organoclay ratio. Polymer 2007; 48:5308-23.

[12] Dennis H, Hunter D, Chang D, Kim S, White J, Cho J, Paul D. Effect of melt processing conditions on the extent of exfoliation in organoclay-based nanocomposites. Polymer 2001; 42:9513-22.

[13] Shah RK, Paul DR. Nylon 6 nanocomposites prepared by a melt mixing masterbatch process. Polymer 2004; 45:2991-3000.

[14] Zhu L, Xanthos M. Effects of process conditions and mixing protocols on structure of extruded polypropylene nanocomposites. J Appl Polym Sci 2004; 93:1891-99.

[15] Lertwimolnun W, Vergnes B. Effect of processing conditions on the formation of polypropylene/organoclay nanocomposites in a twin screw extruder. Polym Eng Sci 2006; 46:314-23.

[16] Lertwimolnun W, Vergnes B. Influence of screw profile and extrusion conditions on the microstructure of polypropylene/organoclay nanocomposites. Polym Eng Sci 2007; 47:2100-09.

[17] Treece M, Zhang W, Moffitt R, Oberhauser J. Twin-screw extrusion of polypropylene-clay nanocomposites: Influence of masterbatch processing, screw rotation mode, and sequence. Polym Eng Sci 2007; 47:898-911.

[18] Médéric P, Aubry T, Razafinimaro T. Structural and rheological properties as a function of mixing energy for polymer/layered silicate nanocomposites. Int Polym Proc 2009; 3:261-66.

[19] Fornes TD, Yoon PJ, Keskkula H, Paul DR. Nylon 6 nanocomposites: the effect of matrix molecular weight. Polymer 2001; 42:9929-40.

[20] Morgan AB, Gilman JW. Characterization of polymer-layered silicate (clay) nanocomposites by transmission electron microscopy and X-ray diffraction: A comparative study. J Appl Polym Sci 2003; 87:1329-38.

[21] Schaefer DW, Justice RS. How nano are nanocomposites? Macromolecules 2007; 40:8501-8517.

[22] Krishnamoorti R, Giannelis EP. Rheology of end-tethered polymer layered silicate nanocomposites. Macromolecules 1997; 30:4097-102.

[23] Ren J, Silva AS, Krishnamoorti R. Linear viscoelasticity of disordered polystyrene-polyisoprene block copolymer based layered silicate nanocomposite. Macromolecules 2000; 33:3739-46.

[24] Solomon MJ, Almusallam AS, Seefeldt KF, Somwangthanaroj A, Varadan P. Rheology of polypropylene/clay hybrid materials. Macromolecules 2001; 34:1864-72.

[25] Aubry T, Razafinimaro T, Médéric P. Rheological investigation of the melt state elastic and yield properties of a polyamide-12 layered silicate nanocomposite. J Rheol 2005; 49:425-40.

[26] Vermant J, Ceccia S, Dolgovskij M, Maffettone P, Macosko C. Quantifying dispersion of layered nanocomposites via melt rheology. J Rheol 2007; 51():429-50.

[27] Cassagnau P. Melt rheology of organoclay and fumed silica nanocomposites. Polymer 2008; 49:2183-96. 
[28] Vergnes, B. The use of apparent yield stress to characterize exfoliation in polymer nanocomposites. Int Polym Proc 2011; 26:229-32.

[29] Zouari R, Domenech T, Vergnes B, Peuvrel-Disdier E. Time evolution of the structure of organoclay/polypropylene nanocomposites and application of the time-temperature superposition principle. J Rheol 2012; 56:725-742.

[30] Domenech T, Peuvrel-Disdier E, Vergnes B. Influence of twin-screw processing conditions on structure and properties of polypropylene - organoclay nanocomposites. Int Polym Proc 2012; in press.

[31] Vergnes B, Della Valle G, Delamare L. A global computer software for polymer flows in corotating twin screw extruders. Polym Eng Sci 1998; 38:1781-92.

[32] Drozdov AD, Høg Lejre AL, deC Christiansen J. Viscoelasticity, viscoplasticity, and creep failure of polypropylene/clay nanocomposites. Compos Sci Technol 2009; 69(15-16):2596-603.

[33] Carneiro OS, Covas JA, Vergnes B. Experimental and theoretical study of the twin screw extrusion of polypropylene. J Appl Polym Sci 2000; 78:1419-30.

[34] Vergnes B, Berzin F. Modelling of flow and chemistry in twin screw extruders. Plast, Rubber, Comp: Macromol Eng 2004; 33:409-15.

[35] Villmow T, Kretzschmar B, Pötschke P. Influence of screw configuration, residence time, and specific mechanical energy in twin-screw extrusion of polycaprolactone/multi-walled carbon nanotube composites. Compos Sci Technol 2010;70:2045-55.

[36] Pujari S, Dougherty L, Mobuchon C, Carreau PJ, Heuzey MC, Burghardt WR. X-ray scattering measurements of particle orientation in a sheared polymer/clay dispersion. Rheol Acta 2010; 50:3-16.

[37] Dykes LMC, Torkelson JM, Burghardt WR. Shear-induced orientation in well-exfoliated polystyrene/clay nanocomposites. Macromolecules 2012; 45:1622-30.

[38] Collin V, Boudimbou I, Peuvrel-Disdier E. New insights in dispersion mechanisms of carbon black in a polymer matrix under shear by rheo-optics. J Appl Polym Sci 2012, DOI: 10.1002/app.37769.

[39] Kasaliwal GR, Pegel S, Göldel A, Pötschke P, Heinrich G. Analysis of agglomerate dispersion mechanisms of multiwalled carbon nanotubes during melt mixing in polycarbonate. Polymer 2010; 51:2708-20.

[40] Scurati A, Feke DL, Manas-Zloczower I. Analysis of the kinetics of agglomerate erosion in simple shear flows. Chem Eng Sci 2005; 60:6564-73.

\section{Table 1}

Twin screw extrusion processing conditions and associated material parameters, where $t_{\text {mean }}$ stands for mean residence time and $T_{\text {melt }}$ for melt temperature at the die exit.

\begin{tabular}{ccccccc}
\hline $\begin{array}{c}N \\
(\mathrm{rpm})\end{array}$ & $\begin{array}{c}Q \\
(\mathrm{~kg} / \mathrm{h})\end{array}$ & $\begin{array}{c}T_{\text {reg }} \\
\left({ }^{\circ} \mathrm{C}\right)\end{array}$ & $\begin{array}{c}S M E \\
(\mathrm{kWh} / \mathrm{t})\end{array}$ & $\begin{array}{c}t_{\text {mean }} \\
(\mathrm{s})\end{array}$ & $\begin{array}{c}T_{\text {melt }} \\
\left({ }^{\circ} \mathrm{C}\right)\end{array}$ & $\begin{array}{c}d_{001} \\
(\mathrm{~nm})\end{array}$ \\
\hline 100 & 3 & 180 & 150 & 170 & 191 & 3.60 \\
300 & 3 & 180 & 398 & 120 & 197 & 3.48 \\
500 & 3 & 180 & 633 & 110 & 211 & 3.31 \\
700 & 3 & 180 & 873 & 98 & 233 & 3.32 \\
900 & 3 & 180 & 1087 & 90 & 253 & 3.21 \\
500 & 6 & 180 & 395 & 60 & 210 & 3.44 \\
500 & 10 & 180 & 290 & 42 & 211 & 3.47 \\
500 & 15 & 180 & 247 & 32 & 212 & 3.53 \\
500 & 20 & 180 & 224 & 26 & 206 & 3.61 \\
300 & 3 & 200 & 342 & 115 & 218 & 3.47 \\
300 & 3 & 220 & 327 & 110 & 235 & 3.41 \\
300 & 3 & 240 & 292 & 111 & 258 & 3.31 \\
\hline
\end{tabular}




\section{Figures and Captions}

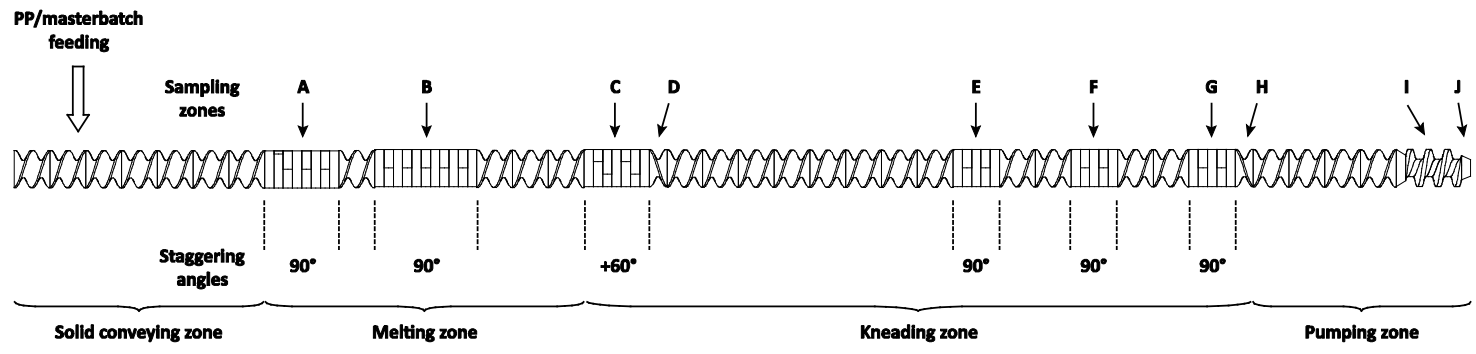

Fig. 1. Screw profile configuration (flow goes from left to right).
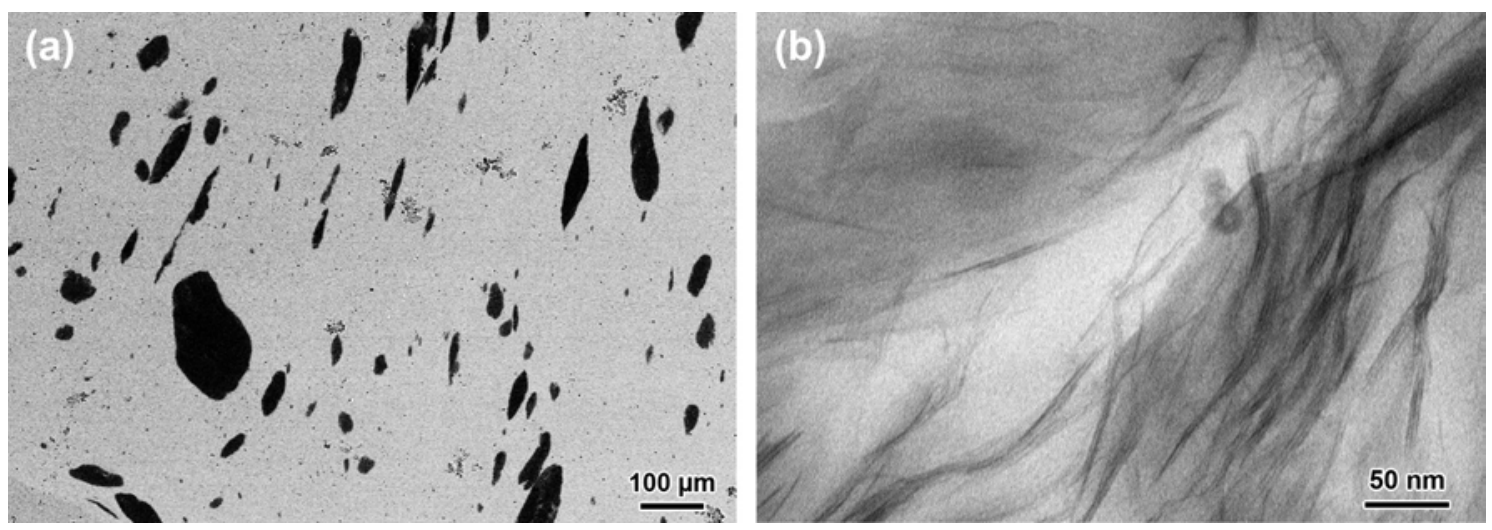

Fig. 2. (a) SEM and (b) TEM pictures of the OMMT masterbatch. 

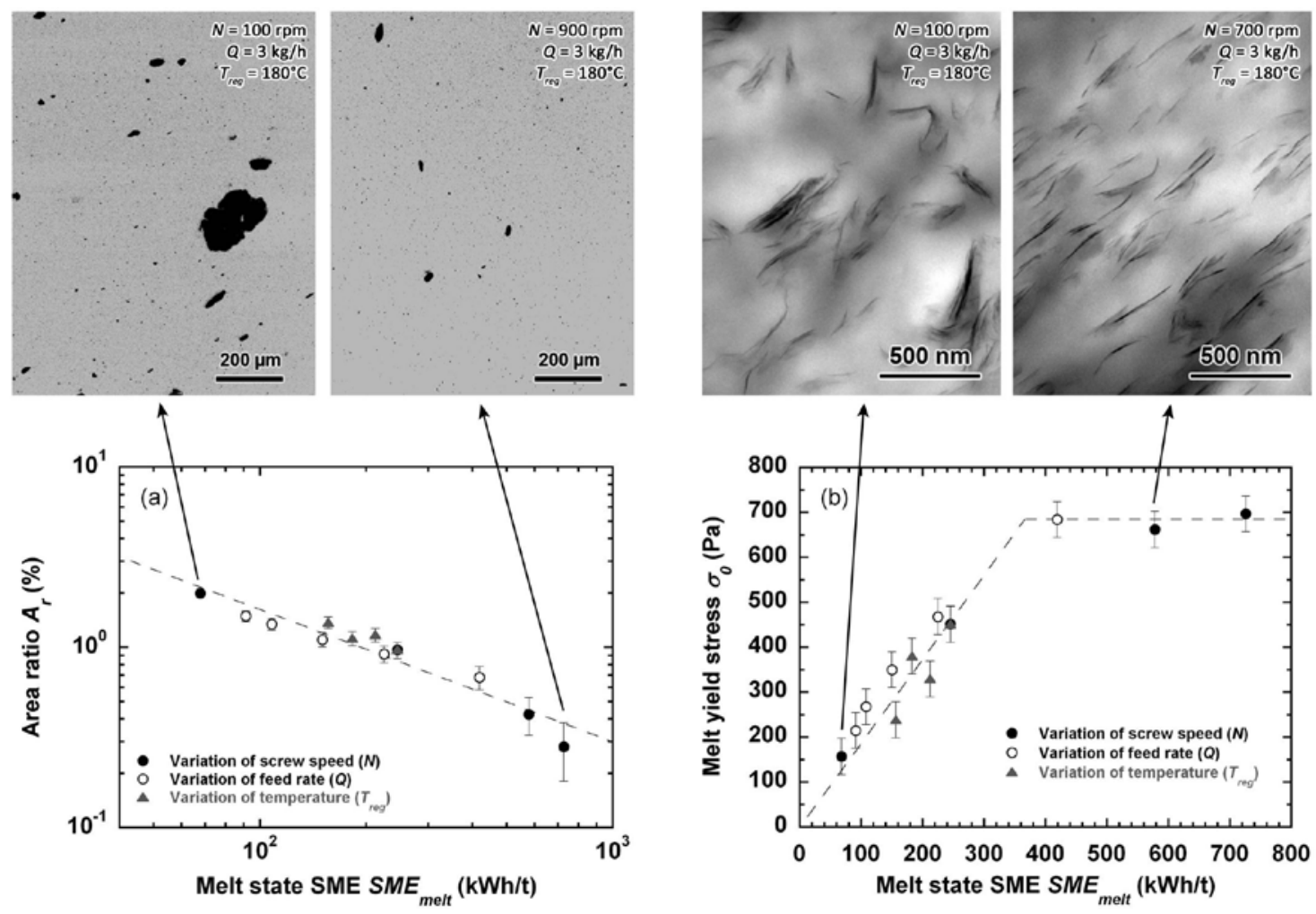

Fig. 3. Evolution of (a) the area ratio and (b) the melt yield stress as a function of $S M E_{\text {melt }}$ for nanocomposites prepared over a wide range of processing conditions, with corresponding SEM and TEM pictures.

(a)

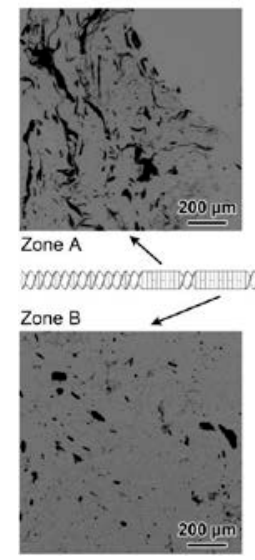

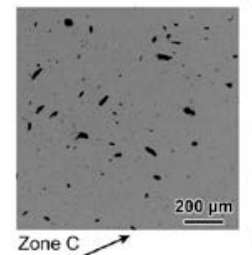

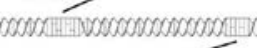

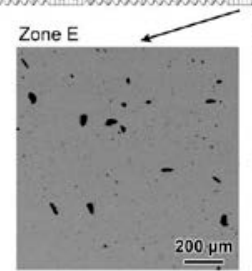

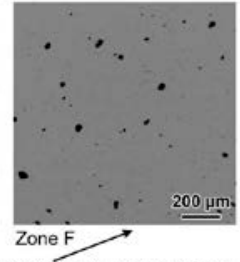

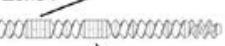

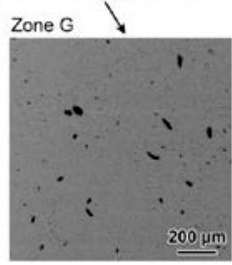

(b)

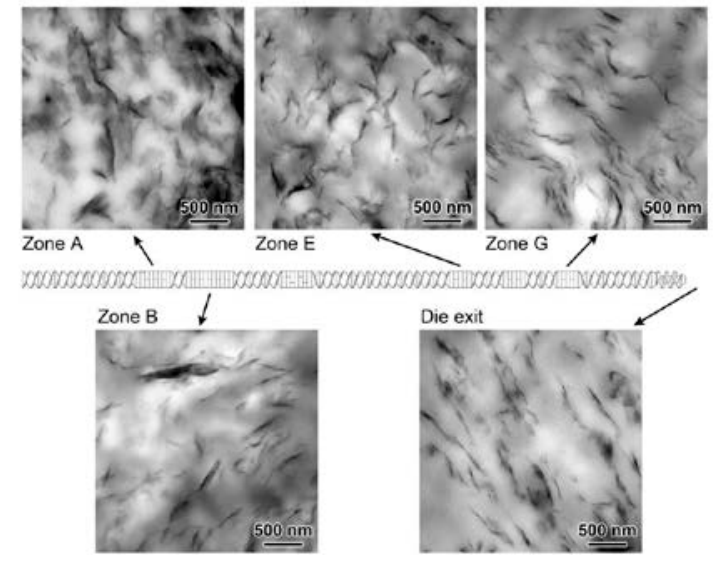

Fig. 4. (a) SEM and (b) TEM pictures of the samples collected along the screw profile after processing at $N=500 \mathrm{rpm}, Q=3 \mathrm{~kg} / \mathrm{h}$ and $T_{\text {reg }}=180^{\circ} \mathrm{C}$. 

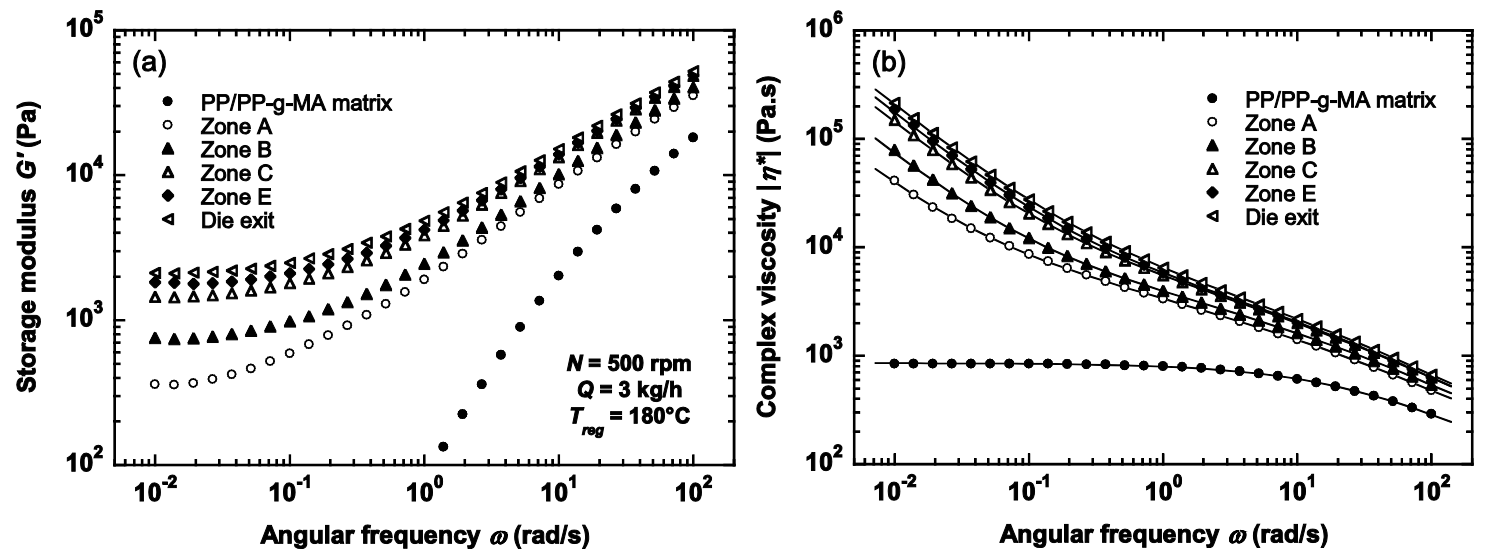

Fig. 5. Frequency sweeps in the linear viscoelastic domain for the PP/PP-g-MA matrix and nanocomposites samples collected along the screw profile after processing at $N=$ $500 \mathrm{rpm}, Q=3 \mathrm{~kg} / \mathrm{h}$ and $T_{\text {reg }}=180^{\circ} \mathrm{C}$ : (a) storage modulus, (b) complex viscosity. Solid lines represent best fits obtained with Eq. (3).
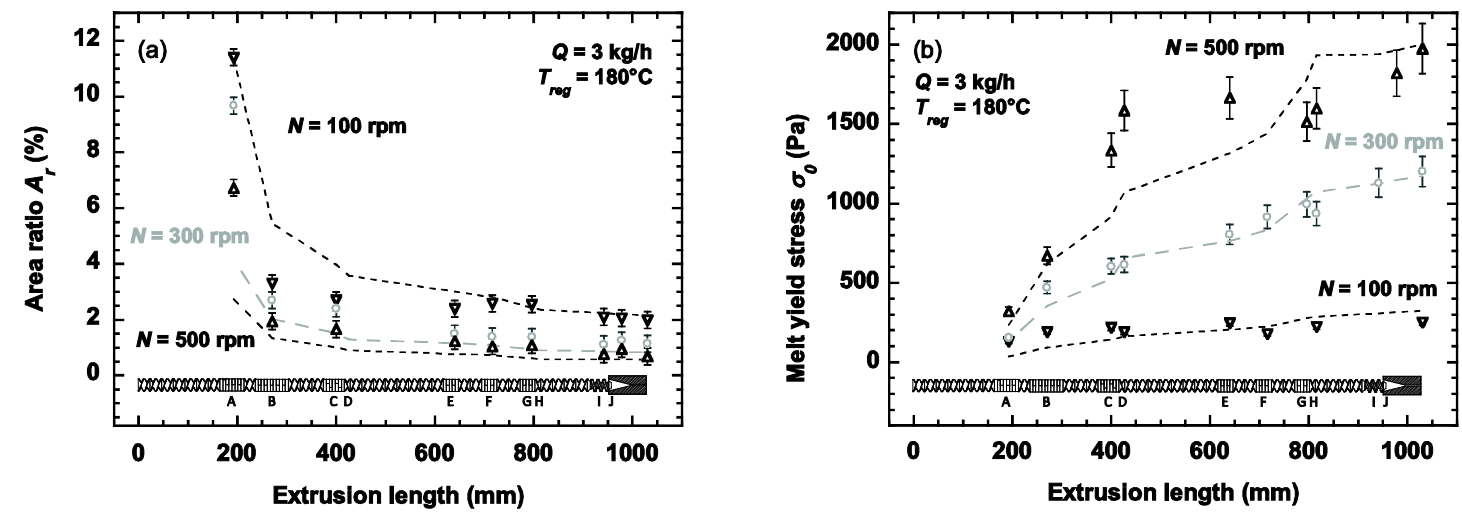

Fig. 6. Evolution of dispersion parameters along the screw profile: (a) area ratio, (b) melt yield stress. Symbols represent experimental data, dashed lines represent simulation results based on Eq. (4) and (5). 


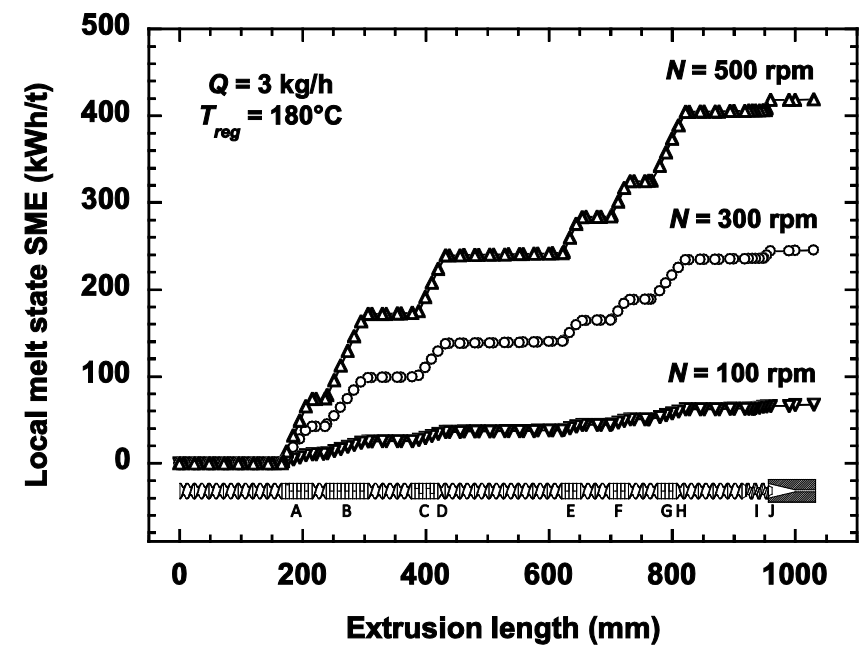

Fig. 7. Evolution of $S M E_{\text {melt }}$ along the screw profile for several screw speeds, obtained from Ludovic ${ }^{\odot}$ calculations.
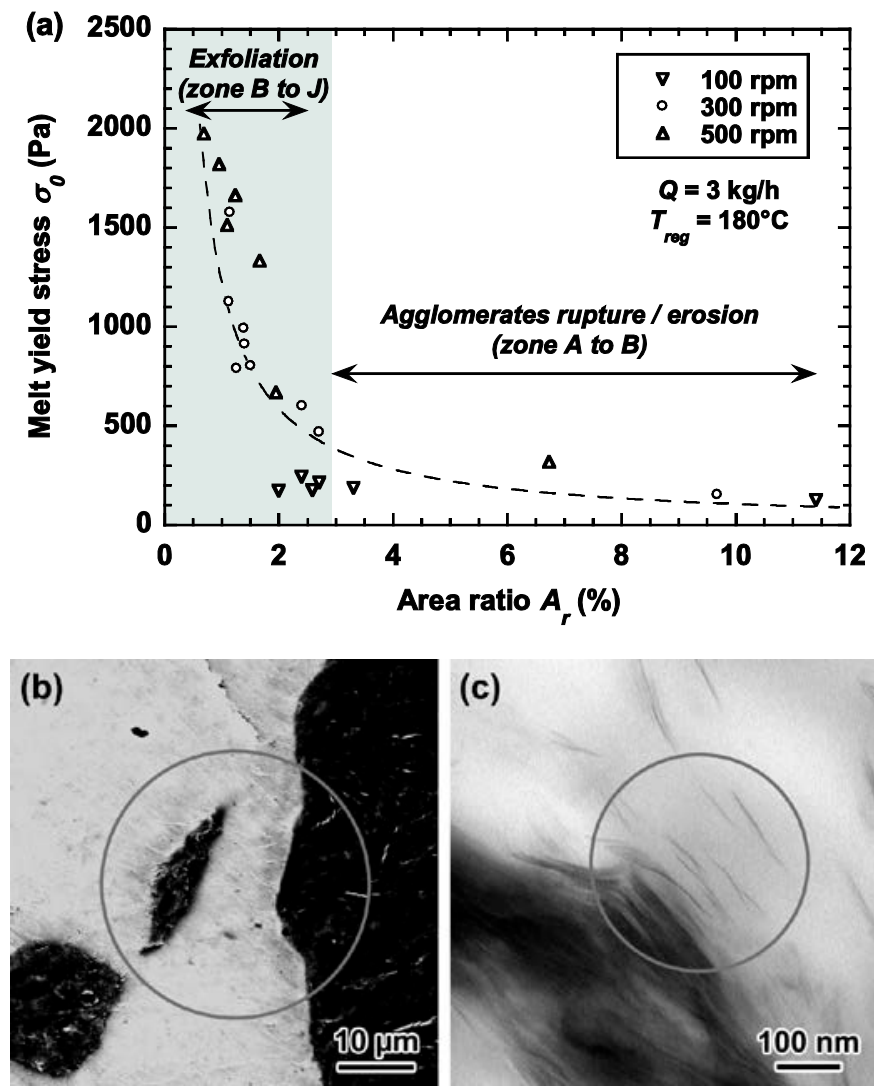

Fig. 8. (a) Dispersion regimes of twin-screw extrusion process, (b) SEM illustration of OMMT agglomerates breakup, (c) TEM illustration of OMMT exfoliation by tactoid peel-off mechanism. 